\title{
Engineering Mode Hybridization in Regular Arrays of Plasmonic Nanoparticles Embedded in 1D Photonic Crystal
}

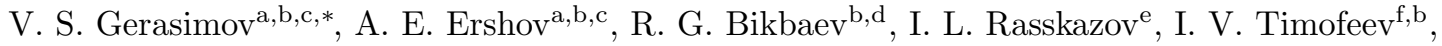 \\ S. P. Polyutov ${ }^{c, b}$, S. V. Karpov ${ }^{b, f, g}$ \\ ${ }^{a}$ Institute of Computational Modeling SB RAS, Krasnoyarsk 660036, Russia \\ ${ }^{b}$ Institute of Nanotechnology, Spectroscopy and Quantum Chemistry, Siberian Federal University, Krasnoyarsk, 660041, \\ Russia \\ ${ }^{c}$ Federal Siberian Research Clinical Centre under FMBA of Russia, Krasnoyarsk, 660037, Russia \\ ${ }^{d}$ Polytechnic Institute, Siberian Federal University, Krasnoyarsk 660041, Russia \\ e The Institute of Optics, University of Rochester, Rochester, NY 14627, USA \\ ${ }^{f}$ Kirensky Institute of Physics, Federal Research Center KSC SB RAS, 660036, Krasnoyarsk, Russia \\ ${ }^{g}$ Siberian State University of Science and Technology, 660014, Krasnoyarsk, Russia
}

\begin{abstract}
We analytically and numerically study coupling mechanisms between $1 \mathrm{D}$ photonic crystal (PhC) and 2D array of plasmonic nanoparticles (NPs) embedded in its defect layer. We introduce general formalism to explain and predict the emergence of $\mathrm{PhC}$-mediated Wood-Rayleigh anomalies, which spectral positions agree well with the results of exact simulations with Finite-Difference Time-Domain (FDTD) method. Electromagnetic coupling between localized surface plasmon resonance (LSPR) and PhC-mediated Wood-Rayleigh anomalies makes it possible to efficiently tailor $\mathrm{PhC}$ modes. The understanding of coupling mechanisms in such hybrid system paves a way for optimal design of sensors, light absorbers, modulators and other types of modern photonic devices with controllable optical properties.
\end{abstract}

Keywords: surface lattice resonance, photonic crystal, optical cavity

\section{Introduction}

Hybrid systems comprised of nanoparticles (NPs) with localized surface plasmon resonance (LSPR) optically coupled to Fabry-Pérot cavities have gained a lot of attention over the last decade [1] due to their exceptional properties which can be used for narrow-band absorption [2, 3, lasers 4, plasmonic loss mitigation [5], electric field enhancement [6], and sensing [7-9. Apart from various practical implementations of Fabry-Pérot cavities, 1D photonic crystal (PhC) with defect layer represents a case of specific interest due to non-trivial coupling of NPs with PhC defect modes. The latter phenomenon may yield in improved performance of surface-enhanced Raman spectroscopy [10, ultrafast light modulation [11, PhC mode splitting [12, 13], and controllable discretization of NPs optical absorption [14].

\footnotetext{
${ }^{*}$ Corresponding author

Email address: gerasimov@icm.krasn.ru (V. S. Gerasimov)
} 
To the day, there is a number of well-established numerical procedures and theoretical models which allow to calculate electromagnetic properties of NPs embedded in half-space [15, 16] or stratified media [17 20]. As a rule of thumb, resonant properties of regular 1D [21, 22] or 2D [23] arrays are significantly suppressed in the presence of the substrate. Though, in the stratified medium, the existence of different coupling scenarios between plasmonic and photonic modes can be exploited in sensors [24, nanoantennas [25], and other applications [26 28].

So-called surface lattice resonances (SLRs) which have gained significant attention during the past decade [29]38] represent the case of specific interest due to their unique properties. Strong coupling between LSPRs and the grating Wood-Rayleigh anomalies [39, 40] in regular arrays of NPs gives rise to remarkably narrow resonances with exceptionally high quality factor. While the most of publications study only general properties of regular NPs arrays embedded in the non-homogeneous environment, the physics behind sophisticated coupling regimes between plasmonic and photonic modes is usually hindered. The understanding of modes coupling in such systems, and the development of analytical models that predict their optical properties represent quite important applied problem.

In this paper, we study hybrid nanostructure comprised of a $1 \mathrm{D} \mathrm{PhC}$ with a defect layer and a $2 \mathrm{D}$ periodic lattice of plasmonic Au nanodisks embedded in it. We reveal different mode hybridization scenarios by varying geometrical parameters of PhC-NPs system: radius of NPs, period of NPs array, and thickness of $\mathrm{PhC}$ defect layer. We introduce theoretical model to predict the position of Wood-Rayleigh anomalies created by $\mathrm{PhC}$, and to reveal possible coupling scenarios in PhC-NPs system.

\section{Methods}

We consider $\mathrm{PhC}$ with unit cell which consists of two layers: silica dioxide $\left(\mathrm{SiO}_{2}\right)$ with thickness $d_{a}=$ $120 \mathrm{~nm}$ and permittivity $\varepsilon_{a}=2.1$, and zirconium dioxide $\left(\mathrm{ZrO}_{2}\right)$ with thickness $d_{b}=70 \mathrm{~nm}$ and permittivity $\varepsilon_{b}=4.16$. We assume that $\mathrm{PhC}$ is comprised of 6 unit cells and the defect layer (with thickness $L$ and permittivity $\left.\varepsilon_{\text {def }}=2.25\right)$ in between, as shown in Fig. 1 17. Regular 2D array of Au nanodisks with period $h$, height $H$ and radius $R$ is embedded in the middle of the $\mathrm{PhC}$ defect layer as shown in Fig. 1 1 . Tabulated values for $\varepsilon$ of $\mathrm{Au}$ [1] have been used in simulations.

The optical properties of described structures have been calculated with commercial Finite-Difference Time-Domain (FDTD) package [42. Nanostructures are illuminated from the top by the plane wave with normal incidence along $z$ axis and polarization along $y$ axis. Transmission $T$ has been calculated at the bottom of the simulation box. Periodic boundary conditions have been applied at the lateral boundaries of the simulation box, while perfectly matched layer (PML) boundary conditions were used on the remaining top and bottom sides. An adaptive mesh has been used to reproduce accurately the nanodisk shape. Although FDTD method is a comprehensive and well-established tool which shows excellent agreement 
(a) PhC+NPs

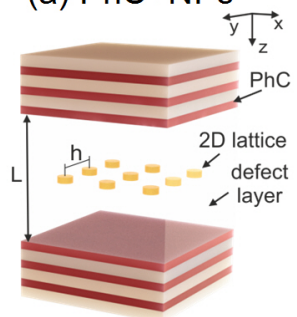

(b) $\mathrm{PhC}$

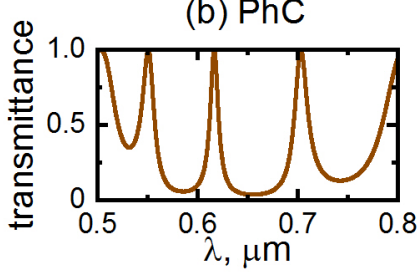

(c) NPs

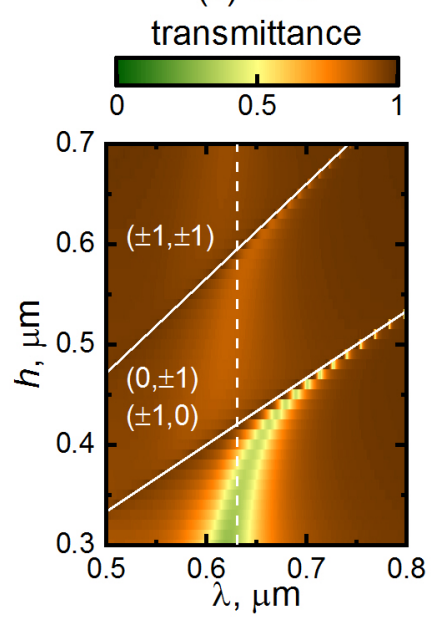

Figure 1: (a) Schematic representation of PhC and 2D array of NPs embedded in its defect layer; (b) Transmittance of bare PhC with $L=1230 \mathrm{~nm}$; (c) Transmittance of 2D lattice of Au nanodisks with height $H=50 \mathrm{~nm}$ and radius $R=40 \mathrm{~nm}$ embedded in homogeneous environment with $\varepsilon_{\text {def }}=2.25$. Solid lines represent Wood-Rayleigh anomalies of $(p, q)$ order, while vertical dashed line indicates the position of dipolar LSPR of the isolated NP.

with experimental results for SLRs [33, 36, 43] and PhCs [44, 45], extensive convergence tests for each set of parameters have been performed to avoid undesired reflections on the PMLs.

\section{Results and Discussion}

\subsection{Bare NPs Array and Bare PhC}

Before going into details, it is instructive to briefly discuss optical response of bare $\mathrm{PhC}$ and regular array of NPs embedded in homogeneous medium separately. The spectral position and transmission coefficient of $\mathrm{PhC}$ modes can be efficiently controlled by varying layer's materials and thicknesses in $\mathrm{PhC}$ [46]. In this paper, $\mathrm{PhC}$ has the band gap from 500 to $820 \mathrm{~nm}$ with 3 distinct modes at 551, 617 and $703 \mathrm{~nm}$, as shown in Fig. 1 $\mathrm{b}$. In what follows, we will refer to modes at 551 and $703 \mathrm{~nm}$ as odd modes, and at $617 \mathrm{~nm}$ as even mode. The use of this terminology is justified by odd and even number of electric field anti-nodes, respectively.

Figure 1. explicitly shows the existence of strong coupling between LSPR and Wood-Rayleigh anomalies [39, 40] in regular 2D array of NPs embedded in homogeneous environment. Such far-field coupling leads to the emergence of high-quality collective resonances [35, 38, with spectral positions close to Wood-Rayleigh anomalies. In the case of normal illumination, the latter can be found with the following equation:

$$
\lambda_{p, q}=h \sqrt{\frac{\varepsilon_{\mathrm{def}}}{p^{2}+q^{2}}},
$$


where $p, q$ are integers which represent the order of the phase difference in $x$ and $y$ directions. Eq. (1) describes condition of constructive interference for particles within the $X O Y$ plane [47. These anomalies of $(0, \pm 1),( \pm 1,0)$ and $( \pm 1, \pm 1)$ orders are shown in Fig. 11. Note that $\lambda$ is the vacuum wavelength.

\subsection{Understanding Mode Hybridization}

In the case of more complicated alignment of NPs array in PhC defect layer, additional Wood-Rayleigh anomalies emerge due to the coupling with $\mathrm{PhC}$. In general case, the wavevector $\mathbf{k}$ in the defect layer reads as:

$$
k^{2}=k_{x}^{2}+k_{y}^{2}+k_{z}^{2}=\varepsilon_{\text {def }}\left(\frac{2 \pi}{\lambda_{p, q, s}}\right)^{2},
$$

where $k_{x, y, z}$ are corresponding components of $\mathbf{k}$. The conditions of constructive interference for $\mathrm{PhC}$ with NPs embedded in the middle of its defect layer, can be found from:

$$
k_{x} h=2 \pi p, \quad k_{y} h=2 \pi q, \quad k_{z} L=2 \pi s-\varphi .
$$

Here $s$ is the integer which denotes the order of the phase difference in $z$ direction. Eq. (3) takes into account the coupling of NPs through the multiple reflections from PhC. Note that for $L \rightarrow \infty$, the coupling between NPs and PhC becomes negligible, and $k_{z}$ vanishes in Eq. (2) and Eq. (3), which yields in Eq. (1) for Wood-Rayleigh anomalies of NPs array embedded in homogeneous medium.

Equation (3) takes into account the phase shift $\varphi$ [48, that occurs due to reflection from the PhC:

$$
\varphi=\arg \left[\frac{C U_{N-1}}{A U_{N-1}-U_{N-2}}\right] .
$$

Here $U_{N}=\sin [(N+1) K \Lambda] / \sin [K \Lambda], K=\arccos [(A+D) / 2] / \Lambda$ is the Bloch wavenumber, $\Lambda=d_{a}+d_{b}$, and $N$ is the number of PhC periods. $A, C$ and $D$ are the elements of the $2 \times 2 A B C D$ complex matrix which relates the amplitudes of plane waves in layer 1 of the unit cell to the corresponding amplitudes for the equivalent layer in the next $\mathrm{PhC}$ unit cell [48].

Although we assume that linearly polarized external radiation impinges normally on the $\mathrm{PhC}$ surface, the light scattered by the NPs in general case has nonzero $k_{x}$ and $k_{y}$. Thus, the normal vector to the surface, the wave vector $k$ and polarization of electric field $\mathbf{E}$ do not lie in the same plane. For this reason, in our case we have to consider both TE and TM polarizations of the electric field scattered by NPs. In this paper, we denote TE polarization as perpendicular to the plane of incidence, while TM is parallel to it.

Thus, for TE mode: 


$$
\begin{aligned}
& A=e^{i k_{a z} d_{a}}\left[\cos k_{b z} d_{b}+\frac{1}{2} i\left(\frac{k_{b z}}{k_{a z}}+\frac{k_{a z}}{k_{b z}}\right) \sin k_{b z} d_{b}\right], \\
& B=e^{-i k_{a z} d_{a}}\left[\frac{1}{2} i\left(\frac{k_{b z}}{k_{a z}}-\frac{k_{a z}}{k_{b z}}\right) \sin k_{b z} d_{b}\right], \\
& C=e^{i k_{a z} d_{a}}\left[-\frac{1}{2} i\left(\frac{k_{b z}}{k_{a z}}-\frac{k_{a z}}{k_{b z}}\right) \sin k_{b z} d_{b}\right], \\
& D=e^{-i k_{a z} d_{a}}\left[\cos k_{b z} d_{b}-\frac{1}{2} i\left(\frac{k_{b z}}{k_{a z}}+\frac{k_{a z}}{k_{b z}}\right) \sin k_{b z} d_{b}\right]
\end{aligned}
$$

and for TM mode:

$$
\begin{aligned}
& A=e^{i k_{a z} d_{a}}\left[\cos k_{b z} d_{b}+\frac{1}{2} i\left(\frac{\varepsilon_{b} k_{a z}}{\varepsilon_{a} k_{b z}}+\frac{\varepsilon_{a} k_{b z}}{\varepsilon_{b} k_{a z}}\right) \sin k_{b z} d_{b}\right], \\
& B=e^{-i k_{a z} d_{a}}\left[\frac{1}{2} i\left(\frac{\varepsilon_{b} k_{a z}}{\varepsilon_{a} k_{b z}}-\frac{\varepsilon_{a} k_{b z}}{\varepsilon_{b} k_{a z}}\right) \sin k_{b z} d_{b}\right], \\
& C=e^{i k_{a z} d_{a}}\left[-\frac{1}{2} i\left(\frac{\varepsilon_{b} k_{a z}}{\varepsilon_{a} k_{b z}}-\frac{\varepsilon_{a} k_{b z}}{\varepsilon_{b} k_{a z}}\right) \sin k_{b z} d_{b}\right], \\
& D=e^{-i k_{a z} d_{a}}\left[\cos k_{b z} d_{b}-\frac{1}{2} i\left(\frac{\varepsilon_{b} k_{a z}}{\varepsilon_{a} k_{b z}}+\frac{\varepsilon_{a} k_{b z}}{\varepsilon_{b} k_{a z}}\right) \sin k_{b z} d_{b}\right] .
\end{aligned}
$$

Here

$$
\begin{aligned}
& k_{a z}=\sqrt{\frac{\varepsilon_{a}}{\varepsilon_{\mathrm{def}}} k_{z}^{2}+\left(\frac{\varepsilon_{a}}{\varepsilon_{\mathrm{def}}}-1\right) k_{x}^{2}+\left(\frac{\varepsilon_{a}}{\varepsilon_{\mathrm{def}}}-1\right) k_{y}^{2}}, \\
& k_{b z}=\sqrt{\frac{\varepsilon_{b}}{\varepsilon_{\mathrm{def}}} k_{z}^{2}+\left(\frac{\varepsilon_{b}}{\varepsilon_{\mathrm{def}}}-1\right) k_{x}^{2}+\left(\frac{\varepsilon_{b}}{\varepsilon_{\mathrm{def}}}-1\right) k_{y}^{2}}
\end{aligned}
$$

are the wave vectors for corresponding layers of $\mathrm{PhC}$.

The numerical solution of Eq. (2) for the given configuration of PhC and array of NPs (which are described in Eq. (3) and Eq. (4p) provides the set of $(p, q, s)$-order Wood-Rayleigh anomalies for both TE and TM polarizations. It should be noticed, that solutions of Eq. (2) are symmetrical with respect to the following permutations and transformations: $p \leftrightarrow q, p \rightarrow-p$, and $q \rightarrow-q$. Thus, for convenience and without losing the generality, we consider $q \geq p \geq 0$. Due to the symmetry of the system, we limit the discussion with non-negative values of $k_{z}$ and $s$. Finally, it should be noticed that the modes with wave vector parallel to the $2 \mathrm{D}$ array (i.e. with $k_{z}=0$ ) and observed in bare NPs array are also preserved in the presence of $\mathrm{PhC}$ and described by Eq.(1). In what follows, we will denote such modes as $(p, q,-)$.

We would like to emphasize that the PhC-mediated interaction between Wood-Rayleigh anomalies of different orders is not taken into account in presented theoretical treatment. Such interaction can be described within the framework of vector-coupled-mode theory [49]. However, as it will be shown below, our formalism predicts positions of Wood-Rayleigh anomalies with satisfying accuracy. In the case of NPs with 
(a) symmetric

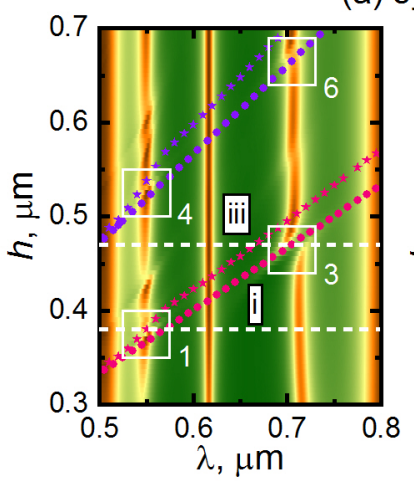

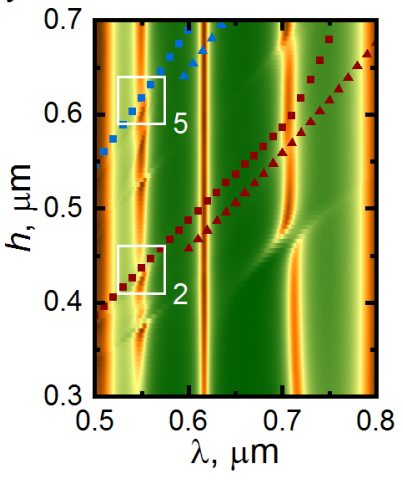

(b) asymmetric

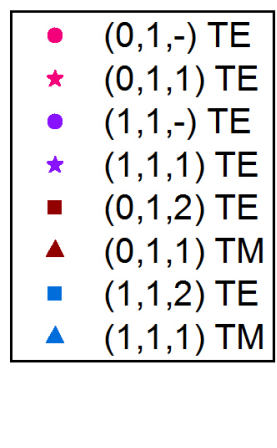

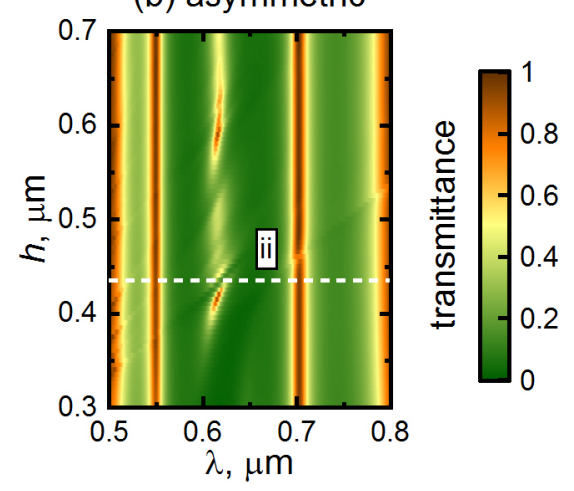

Figure 2: Transmittance of 2D array of gold nanodisks embedded in $\mathrm{PhC}$ defect layer: (a) in the middle, and (b) shifted along $z$ axis by $100 \mathrm{~nm}$. Symbols denote Wood-Rayleigh anomalies of $(p, q, s)$ order for TE and TM polarization. Note that for convenience of the Reader, only position of strongly coupled Wood-Rayleigh anomalies are shown.

height $H$ significantly smaller than the thickness of defect layer $L$, one could ignore the interaction between diffractive modes, though, this approximation has to be used with caution for larger NPs.

\subsection{Coupling Scenarios Between Plasmonic and Photonic Modes}

We start the discussion with NPs arrays embedded in the middle of $\mathrm{PhC}$ defect layer. Fig. 22 represents transmission spectra of this system for different lattice periods $h$. It can be seen that the multiple splittings of $\mathrm{PhC}$ defect modes take place for different $h$ for odd modes only. Interestingly, even mode remains completely intact despite the fact that its frequency almost coincides with the frequency of LSPR. Figure 3 shows detailed insets with regular Wood-Rayleigh anomalies from Fig. 1k and additional anomalies created by coupling of NPs in the array with each other via reflections from PhC. It should be mentioned that anomalies described by Eq. (1) couple with the long wavelength $\mathrm{PhC}$ mode only. It can be explained by strong coupling of LSPR and Wood-Rayleigh anomalies at long wavelength wing of LSPR, which is related to the behavior of dipole sum and inverse dipole polarizability of NP [31. Figures $2 a$ and 3 show that $\mathrm{PhC}$-mediated anomalies couple with short wavelength $\mathrm{PhC}$ mode only. We believe that it is also related to the behavior of the dipole sum and inverse NP polarizability.

The coupling of defect modes with Wood-Rayleigh anomalies can be controlled by moving NP array along $z$ axis. Figure $2 \mathrm{~b}$ demonstrates that in the case of asymmetric alignment of NPs whose positions are shifted by $100 \mathrm{~nm}$ along $z$ axis, even mode strongly couples to NP array, while odd modes exhibit weak coupling. The transmission spectra of the structure for symmetric and asymmetric cases and different $h$ are shown in Fig 2. Variation of the NPs array period $h$ allows to achieve the coupling of any PhC mode with SLR using symmetric geometry for odd PhC modes and asymmetric geometry for even modes. 

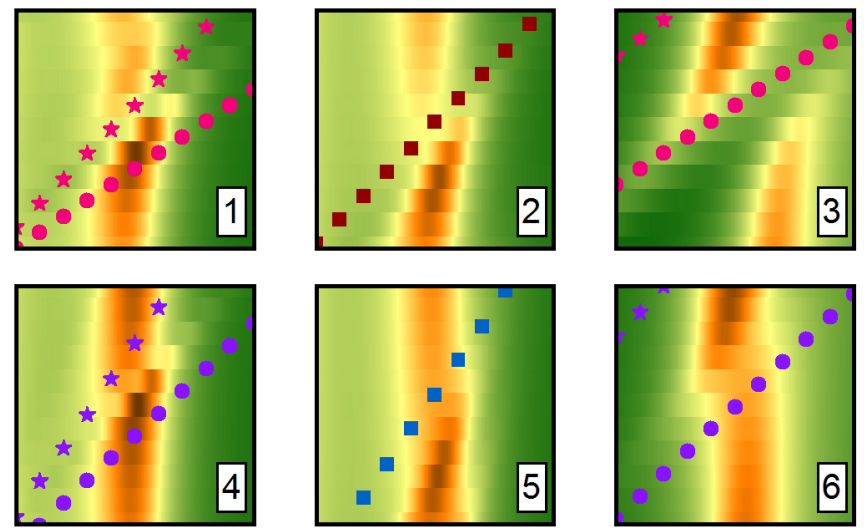

Figure 3: Detailed illustration of various hybridization scenarios for Wood-Rayleigh anomalies shown in Fig. 22a.

The various coupling scenarios for symmetric and asymmetric alignment can be understood as follows. Let us turn to the electric field distribution within the defect layer, which is shown in Fig. 4. In the case of even mode with $\lambda=617 \mathrm{~nm}$, the array is located in the central node of electric field (see Fig. 4a) and the coupling between the defect mode and SLR vanishes. However, for odd modes with $\lambda=551$ and $\lambda=703 \mathrm{~nm}$, the field is localized at the NPs (see Fig $4 \mathrm{~b}, \mathrm{c}, \mathrm{f}, \mathrm{g}$ ) and defect modes are split. The splitting of even modes can be achieved when the NPs array is located at antinode of the electric field, which is easily controlled by shifting the NPs array along $z$ axis. The corresponding field distribution for split defect mode is shown in Fig. 4h,e.

Now let us turn to the investigation of the influence of NPs size and PhC defect layer thickness on optical properties and mode coupling scenarios in plasmonic-photonic system. The transmission spectra of the plasmonic-photonic structure for different values of $R$ and for $h=380 \mathrm{~nm}$ are shown in Fig. 5 a. In this case, splitting of the defect mode at $\lambda=551 \mathrm{~nm}$ is observed. It should be noticed that the splitting of this defect mode doesn't depend on $R$ for $R>40 \mathrm{~nm}$. In this case, the spectral position of the transmission maxima also does not change. This is explained by the fact that the position of the additional WoodRayleigh anomalies doesn't depend on the frequency of localized plasmon resonance. It should be noted that for $R>80 \mathrm{~nm}$, a combined band gap as a superposition of the $\mathrm{PhC}$ band gap and the opacity region of the plasmonic array can be emerged. As a result, the width of the band gap increases roughly by half. At the same time, splitting of the defect mode is not observed when a $\mathrm{PhC}$ is coupled with a two-dimensional lattice of NPs with $R<20 \mathrm{~nm}$. In this case, the wavelength of SLR doesn't coincide with the wavelength of the defect mode, but falls into the frequency interval lying between the $\mathrm{PhC}$ defect modes with $\lambda=617 \mathrm{~nm}$ and $\lambda=703 \mathrm{~nm}$. Finally, the even mode $\lambda=617 \mathrm{~nm}$ remains intact in all cases both for $h=380 \mathrm{~nm}$ and $h=470 \mathrm{~nm}$, as expected from Fig. 2a, while odd mode at $\lambda=703 \mathrm{~nm}$ is significantly suppressed for $R>50 \mathrm{~nm}$ at $h=380 \mathrm{~nm}$ and around $R=35 \mathrm{~nm}$ at $h=470 \mathrm{~nm}$ (see. Fig. 5b). 


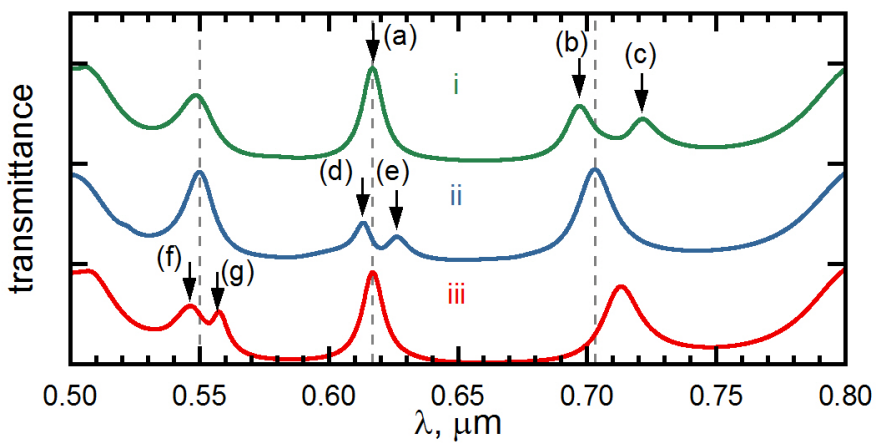

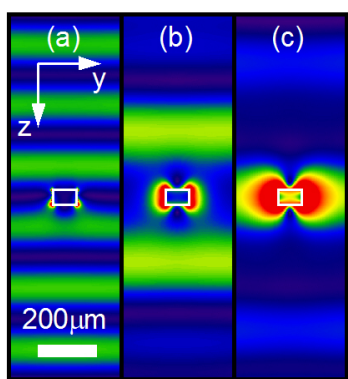

$|\mathbf{E}| / \mathbf{E}_{0} \mid$

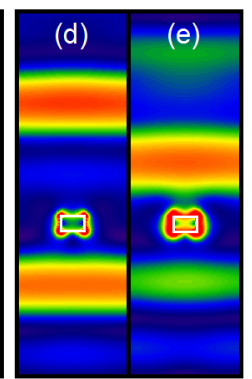

$|\mathbf{E}| / \mathbf{E}_{0} \mid$

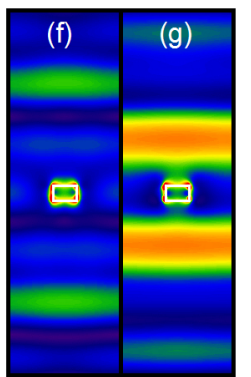

$|\mathrm{E}| /\left|\mathrm{E}_{0}\right|$
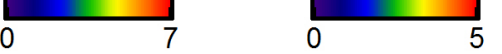

0

Figure 4: (top panel) Transmission spectra for symmetric (i and iii) configuration with $h=470 \mathrm{~nm}$ and $h=380 \mathrm{~nm}$, respectively, and for asymmetric configuration (ii) with $h=435 \mathrm{~nm}$. Corresponding configurations are shown by white dashed lines in Fig. 2 Vertical dashed lines represent positions of PhC modes; (bottom panel) Normalized electric field distribution within the defect layer of PhC with embedded NPs for corresponding alignments (i, ii, and iii) at following wavelengths $\lambda$ : a) $617 \mathrm{~nm}$; b) $697 \mathrm{~nm}$; c) $721 \mathrm{~nm}$; d) $613 \mathrm{~nm}$; e) $626 \mathrm{~nm}$; f) $546 \mathrm{~nm}$; g) $557 \mathrm{~nm}$. White rectangles denote boundaries of nanodisks. Illumination is along $z$ axis, from the top.

The variation of the $\mathrm{PhC}$ defect layer thickness $L$ is also the efficient way to control the spectral properties of PhC-NPs structure. Figure 6a shows that the spectral position of $\mathrm{PhC}$ modes slightly shifts to longer wavelength region with the increasing of $L$. At the same time, the number of $\mathrm{PhC}$ modes also increases from 2 for $L=500 \mathrm{~nm}$ to 4 for $1400 \mathrm{~nm}$. Figure 6b demonstrates that for $L=1060$ and $L=640 \mathrm{~nm}$, the defect modes between $\lambda=600 \mathrm{~nm}$ and $\lambda=700 \mathrm{~nm}$ can be suppressed. This suppression is explained by the fact that the wavelengths of the defect modes coincide with the wavelength of the SLR for these values of $L$.

Figure 6c shows that the splitting of the $\mathrm{PhC}$ modes can be achieved in a wide spectral range for $h=470 \mathrm{~nm}$. Thus, the splitting is observed at $721 \mathrm{~nm}, 632 \mathrm{~nm}$, and $567 \mathrm{~nm}$ for $L=1230 \mathrm{~nm}, L=1050 \mathrm{~nm}$, and $L=530 \mathrm{~nm}$, correspondingly.

\section{Conclusion}

To conclude, we have developed a simple yet comprehensive analytical model to explain the emergence of additional Wood-Rayleigh anomalies in 2D arrays of NPs embedded in the defect layer of $1 \mathrm{D}$ PhC caused 


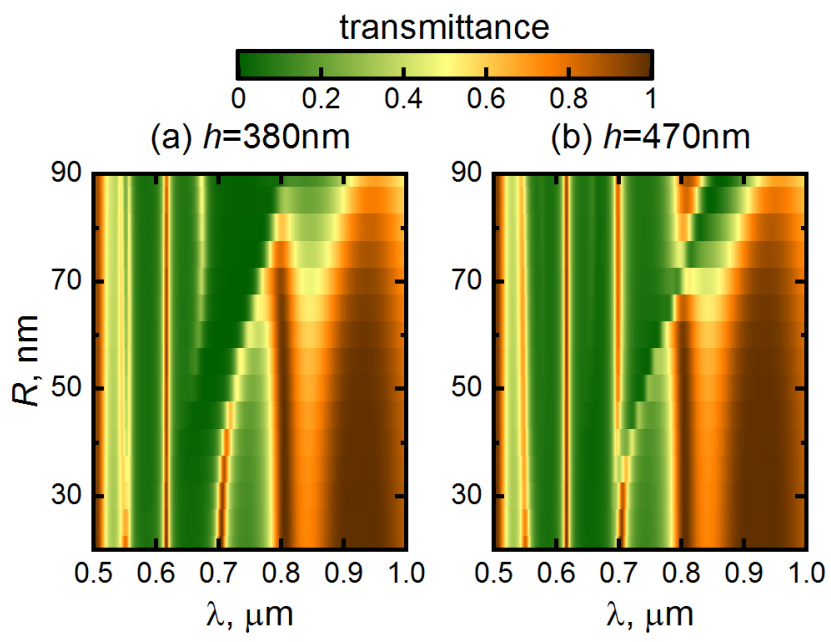

Figure 5: Transmittance of 2D lattice of NPs with (a) $h=380 \mathrm{~nm}$ and (b) $h=470 \mathrm{~nm}$ embedded in the middle of the defect layer of $\mathrm{PhC}$ with $L=1230 \mathrm{~nm}$.

transmittance

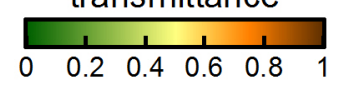

(a) PhC (b) PhC+NPs
$h=380 \mathrm{~nm}$

(c) $\mathrm{PhC}+\mathrm{NPs}$ $h=470 \mathrm{~nm}$
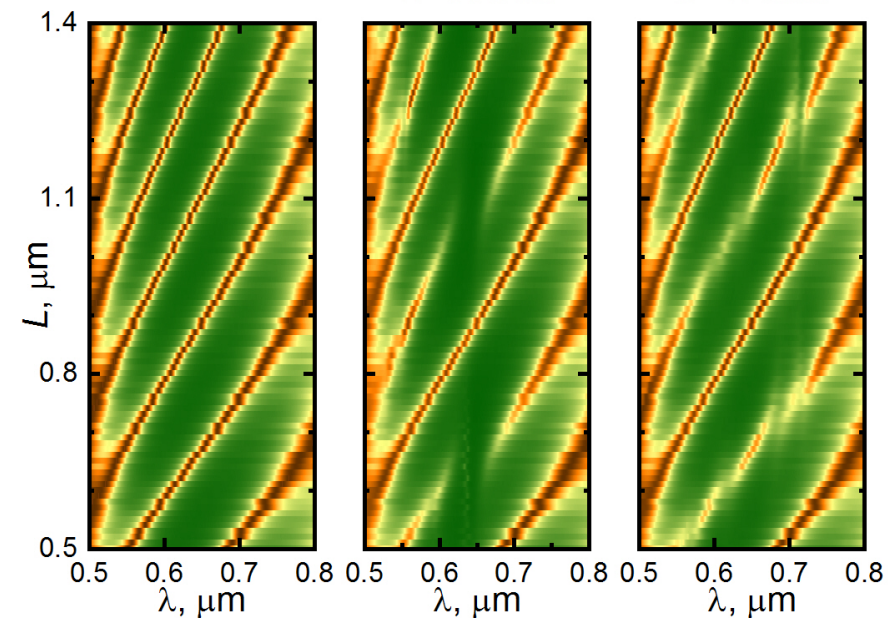

Figure 6: Transmittance of (a) PhC without NPs; and PhC with array of NPs embedded in the middle of its defect layer for two periods $h$ : (b) $380 \mathrm{~nm}$, and (c) $470 \mathrm{~nm}$.

by multiple reflections inside PhC. Non-trivial coupling between LSPRs in arrays of NPs and defect modes of $\mathrm{PhC}$ implies the existence of $\mathrm{PhC}$-mediated Wood-Rayleigh anomalies which spectral positions can be defined within the framework of proposed model with reasonable accuracy. Exact simulations with the FDTD method show excellent agreement between the predicted positions of Wood-Rayleigh anomalies and regions of $\mathrm{PhC}$ modes hybridization. Strong coupling between $\mathrm{PhC}$ and NPs leads to multiple splittings of 
the defect modes which can be tailored by varying period $h$ of NPs array, size of NPs, and PhC defect layer thickness. It was shown that due to the features of electric field distribution within defect layer both even and odd PhC modes can be coupled with SLR by varying the position of NPs array within it. Thus, deeper understanding of modes coupling in hybrid NPs-PhC system paves a way for more efficient control of its optical response for photonics applications which is not easy to achieve with other alternative strategies.

\section{Acknowledgements}

The reported study was funded by Russian Foundation for Basic Research, Government of Krasnoyarsk Territory, Krasnoyarsk Regional Fund of Science (Grant No.18-42-240013); by the RF Ministry of Science and Higher Education, the State contract with Siberian Federal University for scientific research in 2017-2019 (Grant No.3.8896.2017), by the Russian Science Foundation (Project No.18-13-00363) (numerical calculations of Rayleigh anomalies in planar structures and corresponding research).

[1] R. Ameling, H. Giessen, Microcavity plasmonics: strong coupling of photonic cavities and plasmons Laser \& Photonics Reviews 7 (2) (2013) 141-169. doi:10.1002/lpor.201100041 URL http://doi.wiley.com/10.1002/lpor.201100041

[2] D. Chanda, K. Shigeta, T. Truong, E. Lui, A. Mihi, M. Schulmerich, P. V. Braun, R. Bhargava, J. A. Rogers, Coupling of plasmonic and optical cavity modes in quasi-three-dimensional plasmonic crystals, Nature Communications 2 (1) (2011) 477-479. doi:10.1038/ncomms1487 URL http://dx.doi.org/10.1038/ncomms1487

[3] Z. Liu, G. Liu, X. Liu, S. Huang, Y. Wang, P. Pan, M. Liu, Achieving an ultra-narrow multiband light absorption metasurface via coupling with an optical cavity, Nanotechnology 26 (23) (2015) 235702. doi:10.1088/0957-4484/26/23/235702 URL http://iopscience.iop.org/article/10.1088/0957-4484/26/23/235702/pdfhttp://stacks.iop.org/0957-4484/ $26 / i=23 / a=235702$ ?key=crossref $.9986 c 7$ cf38e000d79c26567fa49c4a93

[4] W. Zhou, M. Dridi, J. Y. Suh, C. H. Kim, D. T. Co, M. R. Wasielewski, G. C. Schatz, T. W. Odom, Lasing action in strongly coupled plasmonic nanocavity arrays, Nature Nanotechnology 8 (7) (2013) 506-511. doi:10.1038/nnano.2013.99 URL http://www .nature.com/doifinder/10.1038/nnano.2013.99

[5] R. Kamakura, S. Murai, S. Ishii, T. Nagao, K. Fujita, K. Tanaka, Plasmonic-Photonic Hybrid Modes Excited on a Titanium Nitride Nanoparticle Array in the Visible Region ACS Photonics 4 (4) (2017) 815-822. doi:10.1021/acsphotonics.6b00763

URL http://pubs.acs.org/doi/pdf/10.1021/acsphotonics.6b00763http://pubs.acs.org/doi/abs/10.1021/ acsphotonics.6b00763

[6] S. Alrasheed, E. Di Fabrizio, Effect of Surface Plasmon Coupling to Optical Cavity Modes on the Field Enhancement and Spectral Response of Dimer-Based sensors, Scientific Reports 7 (1) (2017) 10524. doi:10.1038/s41598-017-11140-0 URL http://dx.doi.org/10.1038/s41598-017-11140-0http://www.nature.com/articles/s41598-017-11140-0

[7] M. A. Schmidt, D. Y. Lei, L. Wondraczek, V. Nazabal, S. A. Maier, Hybrid nanoparticle-microcavity-based plasmonic nanosensors with improved detection resolution and extended remote-sensing ability Nature Communications 3 (1) (2012) 1108. doi:10.1038/ncomms2109 URL https://www.nature.com/articles/ncomms2109.pdfhttp://www.nature.com/articles/ncomms2109

[8] M. Bahramipanah, S. Dutta-Gupta, B. Abasahl, O. J. F. Martin, Cavity-Coupled Plasmonic Device with Enhanced 
Sensitivity and Figure-of-Merit, ACS Nano 9 (7) (2015) 7621-7633. doi:10.1021/acsnano.5b02977.

URL www . acsnano.orghttp://pubs .acs.org/doi/10.1021/acsnano.5b02977

[9] J. Chen, Q. Zhang, C. Peng, C. Tang, X. Shen, L. Deng, G.-s. Park, Optical Cavity-Enhanced Localized Surface Plasmon Resonance for High-Quality Sensing IEEE Photonics Technology Letters 30 (8) (2018) 728-731. doi:10.1109/LPT.2018. 2814216

URL http://ieeexplore.ieee.org/document/8310555/

[10] M. Fränzl, S. Moras, O. D. Gordan, D. R. T. Zahn, Interaction of One-Dimensional Photonic Crystals and Metal Nanoparticle Arrays and Its Application for Surface-Enhanced Raman Spectroscopy The Journal of Physical Chemistry C 122 (18) (2018) 10153-10158. doi:10.1021/acs.jpcc.8b02241.

URL http://pubs .acs .org/doi/10.1021/acs .jpcc.8b02241

[11] X. Wang, R. Morea, J. Gonzalo, B. Palpant, Coupling Localized Plasmonic and Photonic Modes Tailors and Boosts Ultrafast Light Modulation by Gold Nanoparticles Nano Letters 15 (4) (2015) 2633-2639. doi:10.1021/acs.nanolett. 5 b00226 URL http://pubs . acs . org/doi/10.1021/acs .nanolett.5b00226

[12] R. Ameling, D. Dregely, H. Giessen, Strong coupling of localized and surface plasmons to microcavity modes Optics Letters 36 (12) (2011) 2218. doi:10.1364/0L.36.002218

URL https://www.osapublishing.org/DirectPDFAccess/897DF6B8-905C-6091-B9EE27D2F68F612F_217316/ ol-36-12-2218.pdf?da=1\&id=217316\&seq=0\&mobile=nohttps : //www . osapublishing.org/abstract.cfm?URI= ol-36-12-2218

[13] S. Y. Vetrov, A. Y. Avdeeva, R. G. Bikbaev, I. V. Timofeev, Traveling of light through a 1D photonic crystal containing a defect layer with resonant dispersion, Optics and Spectroscopy 113 (5) (2012) 517-521. doi:10.1134/S0030400X12110070 URL http://link.springer .com/10.1134/S0030400X12110070

[14] H. Mehrzad, E. Mohajerani, Liquid crystal mediated active nano-plasmonic based on the formation of hybrid plasmonicphotonic modes, Applied Physics Letters 112 (6). doi:10.1063/1.5004076

[15] G. Y. Panasyuk, J. C. Schotland, V. A. Markel, Short-distance expansion for the electromagnetic half-space Green's tensor: general results and an application to radiative lifetime computations. Journal of Physics A: Mathematical and Theoretical 42 (27) (2009) 275203. doi:10.1088/1751-8113/42/27/275203

URL http://stacks . iop.org/1751-8121/42/i=27/a=275203?key=crossref . 45164e4b7f88deefbf837565226b5c64

[16] D. Sikdar, A. A. Kornyshev, Theory of tailorable optical response of two-dimensional arrays of plasmonic nanoparticles at dielectric interfaces. Scientific Reports 6 (1) (2016) 33712. doi:10.1038/srep33712 URL http://dx.doi.org/10.1038/srep33712http://www.nature.com/articles/srep33712

[17] M. Paulus, P. Gay-Balmaz, O. J. F. Martin, Accurate and efficient computation of the Green's tensor for stratified media. Physical Review E 62 (4) (2000) 5797-5807. doi:10.1103/PhysRevE.62.5797 URL https://link.aps.org/doi/10.1103/PhysRevE.62.5797

[18] A. Egel, S. W. Kettlitz, U. Lemmer, Efficient evaluation of Sommerfeld integrals for the optical simulation of many scattering particles in planarly layered media, Journal of the Optical Society of America A 33 (4) (2016) 698. doi:10.1364/JOSAA.33.000698

URL http://dx.doi.org/10.1364/JOSAA.33.000698https://www.osapublishing.org/abstract.cfm?URI= josaa-33-4-698

[19] A. Egel, D. Theobald, Y. Donie, U. Lemmer, G. Gomard, Light scattering by oblate particles near planar interfaces: on the validity of the T-matrix approach Optics Express 24 (22) (2016) 25154. doi:10.1364/0E.24.025154

URL https://www . osapublishing.org/abstract.cfm?URI=oe-24-22-25154

[20] A. Egel, Y. Eremin, T. Wriedt, D. Theobald, U. Lemmer, G. Gomard, Extending the applicability of the T-matrix 
method to light scattering by flat particles on a substrate via truncation of sommerfeld integrals, Journal of Quantitative Spectroscopy and Radiative Transfer 202 (2017) 279-285. doi:10.1016/j.jqsrt.2017.08.016

URL http://linkinghub.elsevier.com/retrieve/pii/S0022407317305939

[21] P. J. Compaijen, V. A. Malyshev, J. Knoester, Surface-mediated light transmission in metal nanoparticle chains, Physical Review B 87 (20) (2013) 205437. doi:10.1103/PhysRevB.87.205437

URL https://link.aps.org/doi/10.1103/PhysRevB.87.205437

[22] I. L. Rasskazov, S. V. Karpov, G. Y. Panasyuk, V. A. Markel, Overcoming the adverse effects of substrate on the waveguiding properties of plasmonic nanoparticle chains Journal of Applied Physics 119 (4). doi:10.1063/1.4940415 URL http://dx.doi.org/10.1063/1.4940415

[23] B. Auguié, X. M. Bendaña, W. L. Barnes, F. J. García de Abajo, Diffractive arrays of gold nanoparticles near an interface: Critical role of the substrate Physical Review B 82 (15) (2010) 155447. doi:10.1103/PhysRevB.82.155447 URL https://link.aps.org/doi/10.1103/PhysRevB.82.155447

[24] A. Vázquez-Guardado, A. Safaei, S. Modak, D. Franklin, D. Chanda, Hybrid Coupling Mechanism in a System Supporting High Order Diffraction, Plasmonic, and Cavity Resonances, Physical Review Letters 113 (26) (2014) 263902. doi:10.1103/PhysRevLett.113.263902

URL https://journals.aps.org/prl/pdf/10.1103/PhysRevLett.113.263902https://link.aps.org/doi/10.1103/ PhysRevLett.113.263902

[25] L. Lin, Y. Zheng, Optimizing plasmonic nanoantennas via coordinated multiple coupling Scientific Reports 5 (2015) 14788. doi:10.1038/srep14788 URL https://www.nature.com/articles/srep14788.pdfhttp://www.nature.com/articles/srep14788

[26] R. Nicolas, G. Lévêque, J. Marae-Djouda, G. Montay, Y. Madi, J. Plain, Z. Herro, M. Kazan, P.-M. Adam, T. Maurer, Plasmonic mode interferences and Fano resonances in Metal-Insulator- Metal nanostructured interface. Scientific Reports 5 (1) (2015) 14419. doi:10.1038/srep14419

URL http://www.pubmedcentral.nih.gov/articlerender.fcgi?artid=4585844\&tool=pmcentrez\&rendertype= abstracthttp://www.nature.com/articles/srep14419

[27] A. N. Shaimanov, K. M. Khabarov, A. M. Merzlikin, I. V. Bykov, A. V. Baryshev, Plasmon resonances in a two-dimensional lattice of metal particles in a dielectric layer: Structural and polarization properties Journal of Experimental and Theoretical Physics 124 (4) (2017) 584-591. doi:10.1134/S1063776117030165

URL http://link.springer.com/10.1134/S1063776117030165

[28] A. Yu, W. Li, Y. Wang, T. Li, Surface lattice resonances based on parallel coupling in metal-insulator-metal stacks, Optics Express 26 (16) (2018) 20695. doi:10.1364/0E.26.020695 URL https://www . osapublishing.org/abstract.cfm?URI=oe-26-16-20695

[29] S. Zou, G. C. Schatz, Narrow plasmonic/photonic extinction and scattering line shapes for one and two dimensional silver nanoparticle arrays, Journal of Chemical Physics 121 (24) (2004) 12606-12612. doi:10.1063/1.1826036

[30] S. Zou, N. Janel, G. C. Schatz, Silver nanoparticle array structures that produce remarkably narrow plasmon lineshapes, Journal of Chemical Physics 120 (23) (2004) 10871-10875. doi:10.1063/1.1760740

[31] V. A. Markel, Divergence of dipole sums and the nature of non-Lorentzian exponentially narrow resonances in onedimensional periodic arrays of nanospheres Journal of Physics B: Atomic, Molecular and Optical Physics 38 (7) (2005) L115-L121. doi:10.1088/0953-4075/38/7/L02

URL http://arxiv.org/abs/physics/0505186\%5Cnhttp://stacks.iop.org/0953-4075/38/i=7/a=L02?key= crossref.c12ad68a9b3ff19f7dae327dfd1ce03a\%5Cnhttp://stacks .iop.org/0953-4075/38/i=7/a=L02?key=crossref . c12ad68a9b3ff19f7dae327dfd1ce03a

[32] B. Auguié, W. L. Barnes, Collective Resonances in Gold Nanoparticle Arrays Physical Review Letters 101 (14) (2008) 
143902. doi:10.1103/PhysRevLett.101.143902

URL https://journals.aps.org/prl/pdf/10.1103/PhysRevLett.101.143902http://link.aps.org/doi/10.1103/ PhysRevLett.101.143902

[33] Y. Chu, E. Schonbrun, T. Yang, K. B. Crozier, Experimental observation of narrow surface plasmon resonances in gold nanoparticle arrays Applied Physics Letters 93 (18) (2008) 181108. doi:10.1063/1.3012365 URL http://aip.scitation.org/doi/10.1063/1.3012365

[34] V. G. Kravets, F. Schedin, A. N. Grigorenko, Extremely Narrow Plasmon Resonances Based on Diffraction Coupling of Localized Plasmons in Arrays of Metallic Nanoparticles. Physical Review Letters 101 (8) (2008) 087403. doi:10.1103/ PhysRevLett.101.087403

URL https://link.aps .org/doi/10.1103/PhysRevLett.101.087403

[35] M. B. Ross, C. A. Mirkin, G. C. Schatz, Optical Properties of One-, Two-, and Three-Dimensional Arrays of Plasmonic Nanostructures. The Journal of Physical Chemistry C 120 (2) (2016) 816-830. doi:10.1021/acs.jpcc.5b10800 URL http://pubs.acs.org/doi/10.1021/acs.jpcc.5b10800http://pubs .acs.org/doi/abs/10.1021/acs.jpcc.5b10800

[36] D. Khlopin, F. Laux, W. P. Wardley, J. Martin, G. A. Wurtz, J. Plain, N. Bonod, A. V. Zayats, W. Dickson, D. Gérard, Lattice modes and plasmonic linewidth engineering in gold and aluminum nanoparticle arrays Journal of the Optical Society of America B 34 (3) (2017) 691. doi:10.1364/JOSAB.34.000691

URL https://www . osapublishing.org/abstract.cfm?URI=josab-34-3-691

[37] V. I. Zakomirnyi, I. L. Rasskazov, V. S. Gerasimov, A. E. Ershov, S. P. Polyutov, S. V. Karpov, Refractory titanium nitride two-dimensional structures with extremely narrow surface lattice resonances at telecommunication wavelengths. Applied Physics Letters 111 (12) (2017) 123107. doi:10.1063/1.5000726 URL http://aip.scitation.org/doi/10.1063/1.5000726

[38] V. G. Kravets, A. V. Kabashin, W. L. Barnes, A. N. Grigorenko, Plasmonic Surface Lattice Resonances: A Review of Properties and Applications, Chemical Reviews 118 (12) (2018) 5912-5951. doi:10.1021/acs.chemrev.8b00243. URL http://pubs .acs.org/doi/10.1021/acs.chemrev.8b00243

[39] R. W. Wood, On a Remarkable Case of Uneven Distribution of Light in a Diffraction Grating Spectrum, Proceedings of the Physical Society of London 18 (1) (1902) 269-275. doi:10.1088/1478-7814/18/1/325 URL http://stacks . iop.org/1478-7814/18/i=1/a=325?key=crossref . 7ebbe1383fb00dffe4b52e1569cdd953

[40] L. Rayleigh, On the Dynamical Theory of Gratings, Proceedings of the Royal Society A: Mathematical, Physical and Engineering Sciences 79 (532) (1907) 399-416. doi:10.1098/rspa.1907.0051 URL http://rspa.royalsocietypublishing.org/cgi/doi/10.1098/rspa.1907.0051

[41] P. B. Johnson, R. W. Christy, Optical Constants of the Noble Metals Physical Review B 6 (12) (1972) $4370-4379$. doi:10.1103/PhysRevB.6.4370 URL https://link.aps.org/doi/10.1103/PhysRevB.6.4370

[42] Lumerical Solutions, "FDTD Solutions" URL www.lumerical.com/tcad-products/fdtd/

[43] B. D. Thackray, P. A. Thomas, G. H. Auton, F. J. Rodriguez, O. P. Marshall, V. G. Kravets, A. N. Grigorenko, SuperNarrow, Extremely High Quality Collective Plasmon Resonances at Telecom Wavelengths and Their Application in a Hybrid Graphene-Plasmonic Modulator Nano Letters 15 (5) (2015) 3519-3523. doi:10.1021/acs.nanolett.5b00930. URL http://pubs.acs.org/doi/pdfplus/10.1021/acs.nanolett.5b00930http://pubs.acs.org/doi/abs/10.1021/acs. nanolett.5b00930

[44] A. Lavrinenko, P. I. Borel, L. H. Frandsen, M. Thorhauge, A. Harpøth, M. Kristensen, T. Niemi, H. M. H. Chong, Comprehensive FDTD modelling of photonic crystal waveguide components, Optics Express 12 (2) (2004) 234 . doi: 10.1364/OPEX.12.000234 
URL https://www .osapublishing.org/oe/abstract.cfm?uri=oe-12-2-234

[45] J. H. E. Kim, L. Chrostowski, E. Bisaillon, D. V. Plant, DBR, Sub-wavelength grating, and Photonic crystal slab FabryPerot cavity design using phase analysis by FDTD Optics Express 15 (16) (2007) 10330. doi:10.1364/0E.15.010330 URL https://www.osapublishing.org/oe/abstract.cfm?uri=oe-15-16-10330

[46] J. J. D. Joannopoulos, S. Johnson, J. N. J. Winn, R. R. D. Meade, Photonic crystals: molding the flow of light, Princeton University Press, 2008. doi:10.1063/1.1586781

URL http://books.google.com/books?hl=en\&lr=\&id=owhE36qiTP8C\&oi=fnd\&pg=PP2\&dq=Photonic+Crystals+Molding+ the+Flow+of+Light\&ots=6XjrrgfTtL\&sig=de1KVRTRbVFyGYHDCOLpWP1xosk\%5Cnhttp://books.google.com/books?id= owhE36qiTP8C\&pgis=1

[47] N. Bonod, J. Neauport, Diffraction gratings: from principles to applications in high-intensity lasers, Advances in Optics and Photonics 8 (1) (2016) 156. doi:10.1364/AOP.8.000156 URL https://www .osapublishing.org/abstract.cfm?URI=aop-8-1-156

[48] P. Yeh, A. Yariv, C.-S. Hong, Electromagnetic propagation in periodic stratified media i general theory, Journal of the Optical Society of America 67 (4) (1977) 423. doi:10.1364/josa.67.000423

[49] P. Paddon, J. F. Young, Two-dimensional vector-coupled-mode theory for textured planar waveguides, Physical Review B 61 (3) (2000) 2090-2101. doi:10.1103/PhysRevB.61.2090

URL https://journals.aps.org/prb/pdf/10.1103/PhysRevB.61.2090https://link.aps.org/doi/10.1103/PhysRevB. 61.2090 